\title{
Molecular alignment from circular dichroic photoelectron angular distributions in $(n+1)$ resonance enhanced multiphoton ionization
}

\author{
Richard L. Dubs \\ Molecular Spectroscopy Division, National Institute of Standards and Technology, Gaithersburg, \\ Maryland 20899 \\ Vincent McKoy \\ Arthur Amos Noyes Laboratory of Chemical Physics, California Institute of Technology, Pasadena, \\ California 91125
}

(Received 22 May 1989; accepted 5 July 1989)

\begin{abstract}
The theory for determination of molecular alignment from circular dichroism in photoelectron angular distributions is generalized to treat the case in which the excitation polarization direction and the laboratory $z$ axis do not coincide. A new method of data analysis is presented here. Alignment created by surface scattering or photofragmentation should be obtainable by these procedures. For studies of orientation with elliptically polarized excitation, differential cross sections at a given collection angle are found to be, to a good approximation, independent of excited-state alignment. Orientation can thus be obtained from differential cross sections by the methods developed by Kummel, Sitz, and Zare [J. Chem. Phys. 88, 6707 (1988)].
\end{abstract}

\section{INTRODUCTION}

The existence of circular dichroism in photoelectron angular distributions (CDAD) from linear molecules fixed in space was first suggested by Cherepkov in $1982 .{ }^{1}$ However, it was not until 1985 that Dubs, Dixit, and McKoy explicitly calculated the first CDAD spectrum from fixed $\mathrm{CO}$ and demonstrated the substantial magnitude of the effect. ${ }^{2,3}$ The large magnitude of the CDAD effect from adsorbed molecules has subsequently been observed experimentally by Westphal et al. ${ }^{4}$ Furthermore, in 1986 Dubs, Dixit, and McKoy showed that CDAD would exist from aligned molecules in the gas phase. ${ }^{5}$ The first CDAD spectra were measured the same year from aligned NO by Appling et al. ${ }^{6}$ Appling et al. later demonstrated several useful features of CDAD in a detailed study of NO optically aligned by onephoton absorption. ${ }^{7}$ Winniczek et al. ${ }^{8}$ have also recently used $(1+1)$ CDAD to determine the alignment of NO produced via photofragmentation of methylnitrite.

The $(n+1)$ CDAD experiment involves $n$-photon excitation of an aligned sample with linearly polarized light followed by single-photon ionization with left and right circularly polarized light. The CDAD spectrum is defined as the difference or asymmetry between the left and right spectra. Photoelectrons are collected in the plane perpendicular to the counterpropagating excitation and ionization lasers. The magnitude and phase of the CDAD spectrum can be related quantitatively to the initial-state alignment. ${ }^{7-11}$

Photoelectron angular distributions are ultimately measured relative to a laboratory $z$ axis. When this axis is defined by the laser polarization direction it is most convenient experimentally to measure the angular distribution with a fixed photoelectron detector and a rotatable laser polarization. Indeed for the $(n+1)$ CDAD experiments performed to date, this configuration has been chosen. ${ }^{6-8}$ The $(n+1)$ CDAD theory has also been developed for this configuration. ${ }^{8,10,11}$ However, it is not hard to imagine alignment experiments in which it is difficult to rotate the laboratory $z$ axis. The alignment of surface-scattered molecules in which the laboratory $z$ axis is defined as the surface normal is one such important example. ${ }^{12}(n+1)$ CDAD theory is generalized here to accommodate these types of experiments. The theory is developed for a fixed laboratory $z$ axis, a fixed detector angle, and a rotatable excitation polarization direction. The theory will also be appropriate for photofragmentation experiments in which the dissociation laser and fragment excitation laser are different.

Kummel et al. ${ }^{13-17}$ have written a series of papers on the determination of initial-state alignment and orientation via $(2+1)$ laser-induced fluorescence (LIF) or $(2+n)$ saturated ionization. The key feature of these experiments is that the total measured signal depends on the excited-state population only and not on its alignment. In the present paper, however, photoionization differential cross sections are examined. These differential cross sections have the inherent property that the excited-state alignment information is intimately entangled with the photoionization dynamics. The extraction of alignment information from differential cross sections is thus complicated. Nevertheless, we present methods by which initial-state alignment can be obtained. The results are cast in the form of linear equations, very similar to those of Kummel et al. ${ }^{13-17}$ but in which the contribution from the photoionization dynamics is apparent. The theory presented here is a generalization of the formalism of Ref. 11 (hereafter referred to as I). Frequent reference and comparison will be made to that work.

To simplify the equations presented here we will sometimes specialize them to an experimental geometry in which the excitation and ionization lasers propagate parallel to the laboratory $y$ axis and photoelectrons are collected in the $x-z$ plane. For surface scattering experiments we choose the surface to be parallel to the $x-y$ plane. In the alignment studies, only equations for samples of cylindrical symmetry are presented. ${ }^{18}$ For orientation measurements we only consider elliptically polarized light with the major axis along the $z$ axis. 


\section{THEORY}

As in I, $(n+1)$ CDAD is treated in two steps: (i) $n$ photon excitation with linearly polarized light to a resonant intermediate state, and (ii) one-photon ionization of the intermediate state with circularly polarized light.

\section{A. $n$-photon excitation to the intermediate state}

Consider one-photon absorption from an initial state with total angular momentum $J^{\prime \prime}$. The excited-state density matrix $\rho_{M_{j}, M_{J}}$ created by one-photon absorption from an initial state with substate populations $N_{M_{J^{\prime \prime}}}$ is

$$
\begin{aligned}
& \rho_{M_{J}, M_{J}}=\sum_{M_{J}} N_{M_{J} \cdot}\left(\begin{array}{ccc}
J^{\prime} & 1 & J^{\prime \prime} \\
-M_{J} & \mu & M_{J^{\prime \prime}}
\end{array}\right) \\
& \times\left(\begin{array}{ccc}
J^{\prime} & 1 & J^{\prime \prime} \\
-M_{J^{\prime}} & \mu^{\prime} & M_{J^{\prime}}
\end{array}\right)\left\langle J^{\prime}\|D\| J^{\prime \prime}\right\rangle^{2} \\
& \times(-1)^{2 J^{\prime}-M_{j}-M_{j}} D_{\mu, \mu_{0}^{\prime}}^{1}(\hat{R}) D_{\mu^{\prime}, \mu_{0}^{\prime}}^{1^{*}}(\hat{R}),
\end{aligned}
$$

where $\left\langle J^{\prime}\|D\| J^{\prime \prime}\right\rangle$ is a reduced matrix element whose value will be set equal to one for now. $D_{M, M}^{L}$, is a rotation matrix, ${ }^{19}$ $\widehat{R}=\left(\theta_{\mathrm{ex}}, \phi_{\mathrm{ex}}\right)$ is the excitation polarization direction measured relative to the laboratory $z$ axis, and $\mu_{0}^{\prime}=0$ for linearly polarized light. The diagonal elements $\rho_{M_{j}, M_{j}}$ are the excited-state substate populations $N_{M_{s}}$. The off-diagonal terms are coherences between the excited-state sublevels which arise because the excitation polarization axis is not directed along the laboratory $z$ axis. If the two axes are collinear $\left(\theta_{\mathrm{ex}}=0\right)$, the second line of Eq. (1) equals unity, $\mu=\mu^{\prime}=0, M_{J^{\prime \prime}}=M_{J^{\prime}}=M_{J}$, and Eq. (1) of I is recovered.

Casting Eq. (1) in terms of alignment parameters, ${ }^{11}$ we have

$$
A_{\mathbf{L} q}=\sum_{L^{\prime \prime}} A_{L^{\prime \prime}} C_{L " \mathbf{L}}^{q}
$$

where $A_{L}$ " are the initial-state alignment parameters and $A_{\mathrm{L} q}$ are the excited-state alignment parameters. For onephoton absorption the $C_{L, L}^{q}$ are given by

$$
\begin{aligned}
C_{L^{\prime \prime} \mathrm{L}}^{q}\left(\theta_{\mathrm{ex}}\right)= & (-1)^{L+1+q+\mu_{0}^{\prime}}\left[\left(2 L^{\prime \prime}+1\right)(2 \mathbf{L}+1)\right]^{1 / 2} \sqrt{4 \pi} \sum_{\mathrm{L}} \sqrt{2 \mathrm{~L}+1} \\
& \times\left\{\begin{array}{ccc}
1 & 1 & \mathrm{~L} \\
J^{\prime} & J^{\prime} & \mathbf{L} \\
J^{\prime \prime} & J^{\prime \prime} & L^{\prime \prime}
\end{array}\right\}\left(\begin{array}{ccc}
1 & 1 & \mathrm{~L} \\
\mu_{0}^{\prime} & -\mu_{0}^{\prime} & 0
\end{array}\right)\left(\begin{array}{ccc}
\mathrm{L} & \mathbf{L} & L^{\prime \prime} \\
q & -q & 0
\end{array}\right) Y_{\mathrm{Lq}}\left(\theta_{\mathrm{ex}}, \phi_{\mathrm{ex}}\right) .
\end{aligned}
$$

For two-photon absorpion the $C_{L^{\prime \prime} \mathrm{L}}^{q}$ are given by

$$
\begin{aligned}
C_{L^{\prime \prime} \mathrm{L}}^{q}\left(\theta_{\mathrm{ex}}\right)= & (-1)^{\mathbf{L}+q}\left[\left(2 L^{\prime \prime}+1\right)(2 \mathbf{L}+1)\right]^{1 / 2} \sqrt{4 \pi} \sum_{\mathrm{L}} \sqrt{2 \mathrm{~L}+1} \\
& \times\left\{\begin{array}{ccc}
2 & 2 & \mathrm{~L} \\
J^{\prime} & J^{\prime} & \mathbf{L} \\
J^{\prime \prime} & J^{\prime \prime} & L^{\prime \prime}
\end{array}\right\}\left(\begin{array}{ccc}
2 & 2 & \mathrm{~L} \\
2 \mu_{0}^{\prime} & -2 \mu_{0}^{\prime} & 0
\end{array}\right)\left(\begin{array}{ccc}
\mathrm{L} & \mathbf{L} & L^{\prime \prime} \\
q & -q & 0
\end{array}\right) Y_{\mathrm{Lq}}\left(\theta_{\mathrm{ex}}, \phi_{\mathrm{ex}}\right) .
\end{aligned}
$$

If $\theta_{\mathrm{ex}}=0$ then $q=0$ and Eqs. (3) and (4) reduce to Eqs. (6) and (7), respectively of I (where the $q=0$ superscript is suppressed). $\phi_{\mathrm{ex}}$ is chosen to be $180^{\circ}$ for this paper.

Equations (3) and (4) exhibit a potential problem for arbitrary excitation direction. In the previous CDAD theory ${ }^{8,11}$ excited-state alignment parameters were determined from CDAD spectra and then related to initial-state alignment parameters by the $q=0$ analog of Eq. (2). However, because $q$ can now take on nonzero values, the number of excited-state alignment parameters is large, even for a small number of initial-state parameters. Furthermore, the contributions of the excited-state parameters to the angular distributions are not necessarily linearly independent. Fortunately, however, the individual excited-state values are not of interest, and, as we now show, the angular distributions can be related directly to the initial-state alignment parameters.

\section{B. Photoionization of the resonant intermediate state}

The differential cross section for photoionization of a resonant intermediate state described by alignment parameters $A_{\mathrm{L} q}$ can be written as

$$
\begin{aligned}
\frac{\partial \sigma}{\partial \Omega_{\hat{k}} \partial \Omega_{\hat{p}}}= & \sum_{\substack{L L^{\prime} M^{\prime} \\
\mathbf{L} q}} A_{\mathbf{L} q} \beta_{L L^{\prime} M^{\prime}}^{\mathbf{L} \mu_{1} q} Y_{L-\left(M^{\prime}+q\right)} \\
& \times\left(\theta_{k}, \phi_{k}\right) Y_{L^{\prime} M^{\prime}}\left(\theta_{p}, \phi_{p}\right),
\end{aligned}
$$

where $\beta$ depends on the photoionization dynamics and $\left(\theta_{k}, \phi_{k}\right)$ is the photoelectron detection direction. $\left(\theta_{p}, \phi_{p}\right)$ is the photon propagation direction for circularly polarized light $\left(\mu_{0}= \pm 1\right)$ or the photon polarization direction for linearly polarized light $\left(\mu_{0}=0\right)$. The expression for $\beta$ is given for $q=0$ by Eq. (15b) of 1 . The general expression for all $q$ is obtained by substituting the general $3-j$ symbol

$$
\left(\begin{array}{ccc}
L & L^{\prime} & \mathbf{L} \\
-\left(M^{\prime}+q\right) & M^{\prime} & q
\end{array}\right)
$$

for the one with $q=0$ in that equation. This $3-j$ symbol contains the complete $q$ dependence of $\beta$. For $q=0$, Eq. (5) reduces to Eq. (14) of I.

\section{CDAD AND INITIAL-STATE ALIGNMENT}

Equation (5) is now analyzed for a CDAD spectrum, i.e., for the difference spectrum between angular distribu- 
tions obtained with left $\left(\mu_{0}=1\right)$ and right $\left(\mu_{0}=-1\right)$ circularly polarized light. Only $L^{\prime}=1$ terms contribute to this spectrum $^{5,11}$ which forces $L=\mathbf{L}$. We specialize Eq. (5) to the experimental geometry discussed earlier, namely $\left(\theta_{p}, \phi_{p}\right)=\left(90^{\circ}, 90^{\circ}\right)$, which requires $M^{\prime}= \pm 1$. Photoelectron collection is restricted to the $\phi_{K}=0^{\circ}$ (or $180^{\circ}$ ) plane. Finally, both the $J^{\prime}$ and $q$ dependences are factored from $\beta$ and Eq. (2) is substituted for $A_{\mathbf{L} q}$. With some simple algebra the CDAD intensity $I_{\mathrm{CDAD}}$ becomes

$$
I_{\mathrm{CDAD}}\left(\theta_{\mathrm{ex}}, \theta_{k}\right)=\sum_{L^{\prime \mathbf{L}}} a_{L^{\prime \prime} \mathbf{L}} G_{L^{\prime \prime} \mathbf{L}}\left(\theta_{\mathrm{ex}}, \theta_{k}\right),
$$

where

$$
a_{L " \mathrm{~L}}=A_{L,} \tilde{\beta}_{\mathrm{L}}
$$

and

$$
\begin{aligned}
G_{L^{\prime \prime} \mathbf{L}}\left(\theta_{\text {ex }}, \theta_{k}\right)= & X_{\mathbf{L}} \sum_{q} C_{L^{\prime \prime} \mathbf{L}}^{q}\left(\theta_{\text {ex }}, 180^{\circ}\right)\left[\left(\begin{array}{ccc}
\mathbf{L} & 1 & \mathbf{L} \\
-(q+1) & 1 & q
\end{array}\right) Y_{\mathbf{L}-(q+1)}\left(\theta_{k}, 180^{\circ}\right)\right. \\
& \left.+\left(\begin{array}{ccc}
\mathbf{L} & 1 & \mathbf{L} \\
-(q-1) & -1 & q
\end{array}\right)\right] Y_{\mathbf{L}-(q-1)}\left(\theta_{k}, 180^{\circ}\right)
\end{aligned}
$$

Here $X_{\mathrm{L}}$ is the $J^{\prime}$ dependence of $\beta$ and is given by Eq. (20) of I. $\tilde{\beta}_{\mathbf{L}}$ is an unknown constan $\mathrm{t}^{20}$ which depends on the photoionization dynamics but is independent of both $J^{\prime}$ and $q$. Note that $\mathbf{L}=0$ does not contribute to CDAD, ${ }^{9,11}$ The $G_{L}$ "L and thus $I_{\mathrm{CDAD}}$ are dependent on $J^{\prime \prime}$ and $J^{\prime}$ through the $C_{L, L}^{q}$. Note that the $G_{L " L}$ are simply numbers which depend on angular momentum factors $(3-j, 6-j, 9-j$ symbols and spherical harmonics) and can be easily calculated for a specified $\theta_{\mathrm{ex}}, \theta_{k}, J^{\prime \prime}, J^{\prime}$.

Equation (6) is the main result of this paper. It presents a simple relationship between the observed CDAD intensity and the initial-state alignment parameters $A_{L}$. Equation (6) is very similar in form to the equations derived by Kummel et al. for LIF [see Eq. (3) of Ref. 13, for example] with one important difference: the presence of $\tilde{\beta}_{\mathrm{L}}$, the $\mathbf{L}$-dependent contribution of the photoionization dynamics to the differential cross section. We will show how this contribution can be eliminated from the data in the next section. Here we emphasize that this simple and compact form in which the photoionization dynamics contribute to Eq. (6) is exactly what makes the determination of the initial-state alignment possible. This form is unique to $\mathrm{CDAD} ;{ }^{21}$ regular photoelectron angular distributions could not be expressed in this simple way. ${ }^{22}$

\section{EXAMPLES}

Consider an initial-state alignment which can be described completely by two alignment parameters, $A_{0}$ and $A_{2}$ (as in the case of one-photon dissociation ${ }^{23}$ ). For $(1+1)$ CDAD we have

$$
\begin{aligned}
I_{\mathrm{CDAD}}\left(\theta_{\mathrm{ex}}, \theta_{k}\right) & =a_{02} G_{02}+a_{22} G_{22}+a_{24} G_{24} \\
& =A_{0} \tilde{\beta}_{2} G_{02}+A_{2} \tilde{\beta}_{2} G_{22}+A_{2} \tilde{\beta}_{4} G_{24} .
\end{aligned}
$$

The limits on $L$ arise from the angular momentum factors in Eq. (3). Equation (9) suggests a simple experiment to determine the initial-state alignment. For a fixed detection angle, $\theta_{k}$, one simply measures the relative $(1+1)$ CDAD signal for three different excitation angles $\theta_{\mathrm{ex}} \cdot{ }^{24} \mathrm{The} G_{L}{ }^{\mathrm{L}}$ for these three measurements can be used to obtain three equations for the coefficients $a_{02}, a_{22}$, and $a_{24}$. After these equations are solved, the ratio

$$
\frac{a_{22}}{a_{02}}=\frac{A_{2}}{A_{0}}
$$

can be related to $A_{0}^{(2)}$, the alignment parameter commonly extracted from LIF experiments, ${ }^{25}$ by Eq. (8) of I. Note that the photoionization dynamics is completely eliminated from the ratio in Eq. (10).

When more than two alignment parameters describe the initial state and/or more than one photon is used for excitation, Eq. (9) has many more terms. For example, consider a $(2+1)$ CDAD experiment on an initial state described by $A_{0}, A_{2}$, and $A_{4}$ :

$$
\begin{aligned}
I_{\mathrm{CDAD}}\left(\theta_{\mathrm{ex}}, \theta_{k}\right)= & a_{02} G_{02}+a_{04} G_{04}+a_{22} G_{22} \\
& +a_{24} G_{24}+a_{26} G_{26}+a_{42} G_{42} \\
& +a_{44} G_{44}+a_{46} G_{46}+a_{48} G_{48} .
\end{aligned}
$$

The limits on $\mathbf{L}$ now arise from the angular momentum factors in Eq. (4). Equation (11) has many more unknowns than we can realistically hope to determine. Fortunately, however, the magnitude of the $\tilde{\beta}_{\mathbf{L}}$ decreases rapidly with increasing $L$ and we can safely ignore terms with $L \geqslant 6$. Equation (11) then becomes

$$
\begin{aligned}
I_{\mathrm{CDAD}}\left(\theta_{\mathrm{ex}}, \theta_{k}\right)= & a_{02} G_{02}+a_{04} G_{04}+a_{22} G_{22} \\
& +a_{24} G_{24}+a_{42} G_{42}+a_{44} G_{44} .
\end{aligned}
$$

Although this equation has six coefficients, they are interrelated and actually only four quantities are unknown: $A_{2} / A_{0}$, $A_{4} / A_{0}, \beta_{4} / \beta_{2}, A_{0} \beta_{2}$. Therefore the equation appears to be soluble with four measurements. Unfortunately, in practice, difficulties arise from linear dependence in the $G_{L}{ }^{\prime} \mathbf{L}$ for a single branch as a function of excitation angle. These difficulties arise because of the limited number of $Y_{L q}$ which contribute to $C_{L{ }^{q} L}^{q}$ in Eq. (4). On the other hand, the $G_{L^{\prime \prime} L}$ for different branches vary quite differently with excitation angle. Equation (12) is still solved then by four relative measurements but with data from two branches, two measurements from each branch. The experiment will require at least one wavelength scan at a given excitation angle to determine the relative intensities of the two branches. In addition, the line strengths $\left\langle J^{\prime}\|D\| J^{\prime \prime}\right\rangle$ (sometimes called $S_{i j}$ ) in Eq. (1) must be included in the $C_{L}^{q}{ }^{\prime}$ of Eqs. (3) and (4). These line strengths are given for NO by Earls ${ }^{26}$ for one-photon excitation and by Halpern, Zacharias, and Wallenstein ${ }^{27}$ for twophoton excitation. 


\section{ORIENTATION}

For excitation with linearly polarized light, the photoelectron angular distributions are only sensitive to initialstate alignment parameters $\left(L^{\prime \prime}=\right.$ even $)$. To determine orientation moments $\left(L^{\prime \prime}=\right.$ odd $)$ elliptically polarized excitation must be used. ${ }^{14,15,17}$ In this case the use of circularly polarized light (left and right) in the ionization step is inconvenient. Fortunately, linearly polarized ionizing light can be used as long as the polarization direction is fixed along the photoelectron collection direction. This detection geometry is called the PINDAD (polarization into detectorangular distribution) configuration and, like CDAD, has convenient properties related to the alignment of the ionized state. ${ }^{8,11}$

The experiment is performed for a fixed collection angle with the elipticity parameter $\beta_{e}$ of the excitation light scanned from $\beta_{e}=+45$ (left circular polarization) to $\beta_{e}=-45$ (right circular polarization). ${ }^{14,15,17}$ The orientation spectrum is defined as the difference spectrum between those obtained with $+\beta_{e}$ and $-\beta_{e}$ values. This spectrum will be sensitive to the orientation moments ( $L^{\prime \prime}=$ odd) but not the alignment moments $\left(L^{\prime \prime}=\right.$ even $)$ in the initial state. $^{14,15,17}$ The differential cross section $I_{\text {ORIENT }}$ can be cast in a form similar to Eq. (6):

$$
I_{\text {ORIENT }}\left(\beta_{e}, \theta_{k}\right)=\sum_{\substack{L^{*} \mathbf{L} \\ q}} A_{L^{\prime \prime} q \pm} \beta_{\mathbf{L}} G_{L^{\prime \prime} \mathbf{L}}^{q \pm}\left(\beta_{e}, \theta_{K}\right) .
$$

The $A_{L " ~} q_{ \pm}$are the real orientation moments of the initial state. ${ }^{14,15,17}$

For a known, assumed orientation, we were unsuccessful in extracting the orientation moments using Eq. (13), even for multiple excitation branches. As with Eq. (12), the problem arises in the very small number of linearly independent angular functions which contribute to the difference spectrum $I_{\text {ORIENT }}$ through the analog of Eq. (4) for elliptically polarized light. However, we found that the shape of the $I_{\text {ORIENT }}$ spectrum as a function of $\beta_{e}$ was independent of collection angle. This result suggested that to a very good approximation we can assume that only the $\mathbf{L}=0$ terms contributed to Eq. (13), i.e., the differential cross section at a fixed collection angle was proportional to the excited-state population only and not its alignment. With this assumption, the formalism for data analysis has been worked out in extensive detail by Kummel, Sitz, and Zare ${ }^{14}$ and need not be repeated here. We found that the orientation moments extracted from our calculated spectra, using data points from two branches, had an error of $2 \%-8 \%$ with the best results for a collection angle of $0^{\circ}$. Such an error should be acceptable for many applications.

\section{ACKNOWLEDGMENTS}

On of us (R.L.D.) gratefully acknowledges support under a National Research Council Resident Research Associateship. This work was supported by the National Science Foundation (Grant No. CHE-8521391), Air Force Office of Scientific Research (Contract No. 87-0039), and the Office of Health and Environmental Research of the U.S. Department of Energy.

'N. A. Cherepkov, Chem. Phys. Lett. 87, 344 (1982).

${ }^{2}$ R. L. Dubs, S. N. Dixit, and V. McKoy, Phys. Rev. Lett. 54, 1249 (1985).

${ }^{3}$ R. L. Dubs, S. N. Dixit, and V. McKoy, Phys. Rev. B 32, 8389 (1985).

${ }^{4}$ C. Westphal, J. Bansmann, M. Getzlaff, and G. Schönhense, Phys. Rev. Lett. 63, 151 (1989).

${ }^{5}$ R. L. Dubs, S. N. Dixit, and V. McKoy, J. Chem. Phys. 85, 656 (1986).

'J. R. Appling, M. G. White, T. M. Orlando, and S. L. Anderson, J. Chem. Phys. 85, 6803 (1986).

'J. R. Appling, M. G. White, R. L. Dubs, S. N. Dixit, and V. McKoy, J. Chem. Phys. 87, 6927 (1987).

J. W. Winniczek, R. L. Dubs, J: R. Appling, V. McKoy, and M. G. White, J. Chem. Phys. 90, 949 (1989).

${ }^{9}$ R. L. Dubs, S. N. Dixit, and V. McKoy, J. Chem. Phys. 85, 6267 (1986).

${ }^{10}$ R. L. Dubs, S. N. Dixit, V. McKoy, J. Chem. Phys. 86, 5886 (1987).

"'R. L. Dubs, S. N. Dixit, V. McKoy, J. Chem. Phys. 88, 968 (1988).

${ }^{12}$ A. C. Luntz, A. W. Kleyn, and D. J. Auerbach, Phys. Rev. B 25, 4273 (1988).

${ }^{13}$ A. C. Kummel, G. O. Sitz, and R. N. Zare, J. Chem. Phys. 85, 6874 (1986).

${ }^{14}$ A. C. Kummel, G. O. Sitz, and R. N. Zare, J. Chem. Phys. 88, 6707 (1988)

${ }^{15}$ G. O. Sitz, A. C. Kummel, and R. N. Zare, J. Chem. Phys. 87, 3247 (1987)

${ }^{16}$ G. O. Sitz, A. C. Kummel, and R. N. Zare, J. Chem. Phys. 89, 2558 (1988).

${ }^{17}$ G. O. Sitz, A. C. Kummel, and R. N. Zare, J. Chem. Phys. 89, 2572 (1988).

${ }^{18}$ Even for surface scattering experiments, results indicate that cylindrical symmetry is often preserved. See Ref. 16.

${ }^{19}$ M. E. Rose, Elementary Theory of Angular Momentum (Wiley, New York, 1957).

${ }^{20} \tilde{\beta}_{1}$ is related to that of Eq. (19) of I by a simple factor which is irrelevant here.

21 Another technique called PINDAD in I will also have an equation of this form, but the contribution of $L=0$ terms makes extraction of alignment information impractical in this case.

22The contribution to regular angular distributions by $L \neq \mathbf{L}$ terms in Eq. (5) has discouraged the use of differential cross sections for alignment determination. See I.

${ }^{23}$ C. H. Greene and R. N. Zare, Annu. Rev. Phys. Chem. 33, 119 (1982).

${ }^{24}$ As pointed out in Ref. 16, it is better to overdetermine the system with more than three measurements and then perform a least-squares fit of the data to Eq. (9).

${ }^{25}$ C. H. Greene and R. N. Zare, J. Chem. Phys. 78, 6741 (1983).

${ }^{26}$ L. T. Earls, Phys. Rev. 48, 423 (1935).

${ }^{27}$ J. B. Halpern, H. Zacharias, and R. Wallenstein, J. Mol. Spectrosc. 79, 1 (1980). 